\title{
Results of high heat flux qualification tests of W monoblock components for WEST
}

\author{
H Greuner $^{\mathrm{a}, *}$, B Böswirth ${ }^{\mathrm{a}}$, M Lipa ${ }^{\mathrm{b}}$, M Missirlian ${ }^{\mathrm{b}}$ and M Richou ${ }^{\mathrm{b}}$ \\ ${ }^{a}$ Max Planck Institute for Plasma Physics, Boltzmann Str. 2, D-85748 Garching, Germany \\ ${ }^{b}$ CEA, IRFM, F-13108 Saint-Paul-Lez-Durance, France
}

Corresponding author: henri.greuner@ipp.mpg.de

\begin{abstract}
One goal of the WEST project (W Environment in Steady-state Tokamak) is the manufacturing, quality assessment and operation of ITER-like actively water-cooled divertor plasma facing components made of tungsten. Six W monoblock plasma facing units (PFUs) of different suppliers have been successfully evaluated in the high heat flux test facility GLADIS at IPP. Each PFU is equipped with $35 \mathrm{~W}$ monoblocks of an ITER like geometry. However, the $\mathrm{W}$ blocks are made of different tungsten grades and the suppliers applied different bonding techniques between tungsten and the inserted Cu-alloy cooling tubes.

The intention of the HHF test campaign was to assess the manufacturing quality of the PFUs on the basis of a statistical analysis of the surface temperature evolution of the individual W monoblocks during thermal loading with 100 cycles at $10 \mathrm{MW} / \mathrm{m}^{2}$. These tests confirm the non-destructive examinations performed by the manufacturer and CEA prior to the installation of the WEST platform, no defects of the components were detected.
\end{abstract}

Keywords: Tungsten, divertor, Plasma-facing components, WEST, ITER 


\section{Introduction}

One goal of the WEST project (W Environment in Steady-state Tokamak) is the manufacturing, quality assessment and operation of ITER-like actively water-cooled divertor plasma facing components made of tungsten (W). The manufacturing of a significant industrial series of such $\mathrm{W}$ plasma-facing units (PFUs) will contribute to the ITER divertor manufacturing risk mitigation [1].Details of design, operating conditions and arrangement of the 480 "ITER like” PFUs, which will be installed in WEST, are given in reference [2].

An essential contribution is the development of a high heat flux (HHF) test method as a nondestructive assessment of the delivered quality of the PFUs. Only HHF loading can generate thermomechanical stress in the component similar to the expected operating conditions in a fusion device. Therefore the assessment of the delivered components requires HHF tests complementary to the nondestructive examinations (NDE) and quality assessment employed at the manufacturer. Other NDE like ultrasonic-, x-ray inspection or thermography methods do not consider the complex thermomechanical stress situation during heat loading.

In the framework of a scientific collaboration between CEA and IPP, six WEST PFUs of different suppliers have been HHF tested prior to the installation in the WEST platform. The components were evaluated in the HHF test facility GLADIS at IPP [3]. Each PFU has outer dimensions of about $612 \times$ $32 \times 42 \mathrm{~mm}^{3}$ and is equipped with $35 \mathrm{~W}$ monoblocks $\left(12 \mathrm{x} \sim 32 \times 26 \mathrm{~mm}^{3}\right)$. Fig. 1 shows the delivered PFUs. However, the blocks are made of different tungsten grades and the suppliers applied different bonding techniques between tungsten and the inserted $\mathrm{Cu}$-alloy (CuCrZr) cooling tubes.

The paper presents the results of the non-destructive low cycle fatigue tests at $10 \mathrm{MW} / \mathrm{m}^{2}$ and $10 \mathrm{~s}$ loading. During this time the component reaches the thermal equilibrium and the thermo-mechanical stress is fully developed. The $60 \mathrm{~s}$ duty cycle ensures the complete cooling down. We discuss a statistical approach of the surface temperature development during the applied 100 cycles to get further information about the manufacturing quality and the expected life time.

\section{Manufacturing of WEST divertor PFUs}

The procurement of the WEST divertor PFUs, using the ITER W monoblock technology, is ongoing in collaboration with the potential suppliers of the ITER Domestic Agencies (F4E (Europe) and JADA (Japan) in charge of providing ITER divertor vertical targets. The Institute of Plasma Physics, Chinese Academy of Sciences (ASIPP) is also a manufacturing partner in the WEST project. Before launching the series production, six of seven PFUs prototypes, provided by ASIPP and JADA in 2016, are presently installed in WEST during first operation.

\subsection{Components supplied by ASIPP}


Four prototypes tested in GLADIS were manufactured by Advanced Technology \& Materials Co. (AT\&M, China), using hot isostatic pressing (HIP) for cladding oxygen free $\mathrm{Cu}$ to the inner drilling of the W monoblocks. In a next step, HIP is applied for the bonding of the Cu clad onto the CuCrZr tube. The manufacturing process for $\mathrm{W}$-materials (rolled plates), produced by AT\&M, is based on the following main steps: Sintering at $\sim 2000^{\circ} \mathrm{C}$, warm rolling at $\sim 1100^{\circ} \mathrm{C}$, reduction in thickness of about $70 \%$ followed by an annealing. The components are named WCAT-001 - 004 in this paper.

\subsection{Components supplied by JADA}

The prototypes tested in GLADIS were manufactured by two different manufacturers (WJMI-002 by Mitsubishi Heavy Industries (MHI) and WJKA-004 by Kawasaki Heavy Industries (KHI)). Each manufacturer has adopted his own bonding technologies for $\mathrm{W}$-monoblocks with pure $\mathrm{Cu}$ interlayer such as HIP at high temperature $\left(\sim 1000^{\circ} \mathrm{C}, 120 \mathrm{MPa}\right)$ for $\mathrm{KHI}$ and direct casting for MHI. Both suppliers use high temperature brazing with Nicuman 37 brazing filler for the bonding of the W/Cu interface to the CuCrZr tube. Brazing at $\sim 980^{\circ} \mathrm{C}$ serves also as solution annealing of CuCrZr. Gas quenching and the ageing at $480^{\circ} \mathrm{C}$ followed at the end of the heat treatment. AT\&M and A.L.M.T Corp. (Japan) supplied the W-blocks (rolled plates) for MHI and KHI, respectively.

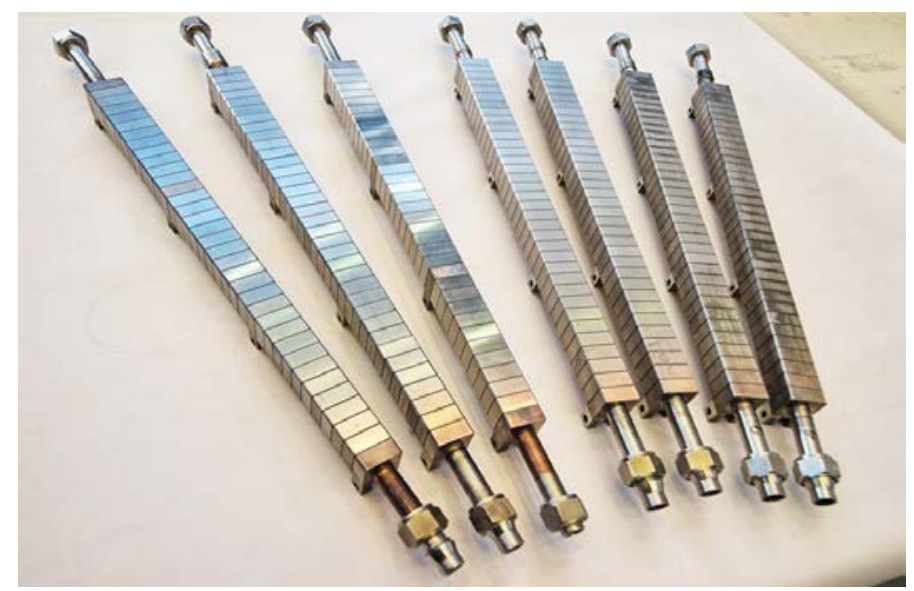

Fig. 1 The image shows the WEST divertor PFUs prototypes. Six of them were HHF tested in GLADIS. Each of them are about $0.6 \mathrm{~m}$ long PFU and made of $35 \mathrm{~W}$ monoblocks bonded on a CuCrZr cooling tube with $\varnothing 12 \mathrm{~mm}$ inner diameter equipped with a swirl as turbulence promoter.

\section{High heat flux loading}

\subsection{Loading and cooling conditions}


The applied cooling conditions ensure a heat transfer similar to the WEST operation requirements: 10 $\mathrm{m} / \mathrm{s}$ water velocity, $\sim 20^{\circ} \mathrm{C}$ inlet temperature and $1 \mathrm{MPa}$ static pressure. (Designed water cooling conditions during operation in WEST: $70^{\circ} \mathrm{C}$ inlet, $2.5 \mathrm{MPa}$ pressure, $10 \mathrm{~m} / \mathrm{s}$ axial velocity [2]. ITER: $70^{\circ} \mathrm{C}$ inlet, 4.0 MPa pressure, $10 \mathrm{~m} / \mathrm{s}$ nominal velocity [4].)

The comparison between the calorimetrically measured absorbed power of the component and the calculated incident beam power is in an agreement better than $\pm 5 \%$ according to the comparison method described in reference [5].

\subsection{High heat flux test procedure}

Four PFUs made by AT\&M were completely assessed with 100 cycles at $10 \mathrm{MW} / \mathrm{m}^{2}$ on 24 blocks of each component. A reduced program of only 10 cycles on each block was carried out for the remaining two components (WJKA-004, WJMI-002) due to time restriction. Despite the fact that only a small part of the component will be exposed to heat fluxes $\geq 10 \mathrm{MW} / \mathrm{m}^{2}$ in the strike points regions of WEST, we have performed the $10 \mathrm{MW} / \mathrm{m}^{2}$ loading on all W blocks, except the end blocks 35 - 34 and $10-1$, respectively. These blocks were loaded with reduced heat flux to avoid an overheating of the cooling tubes and the stainless steel water connectors at the end of the components. Due to the Gaussian heat flux profile with a $10 \pm 0.5 \mathrm{MW} / \mathrm{m}^{2}$ central spot diameter of $60 \mathrm{~mm}$ it was necessary to move the component after each 100 cycles to the next position. The surface temperature evolution of the five central blocks was assessed during the applied 100 cycles of each position. We have performed this procedure at 5 consecutive positions marked by the arrows in Fig. 2.

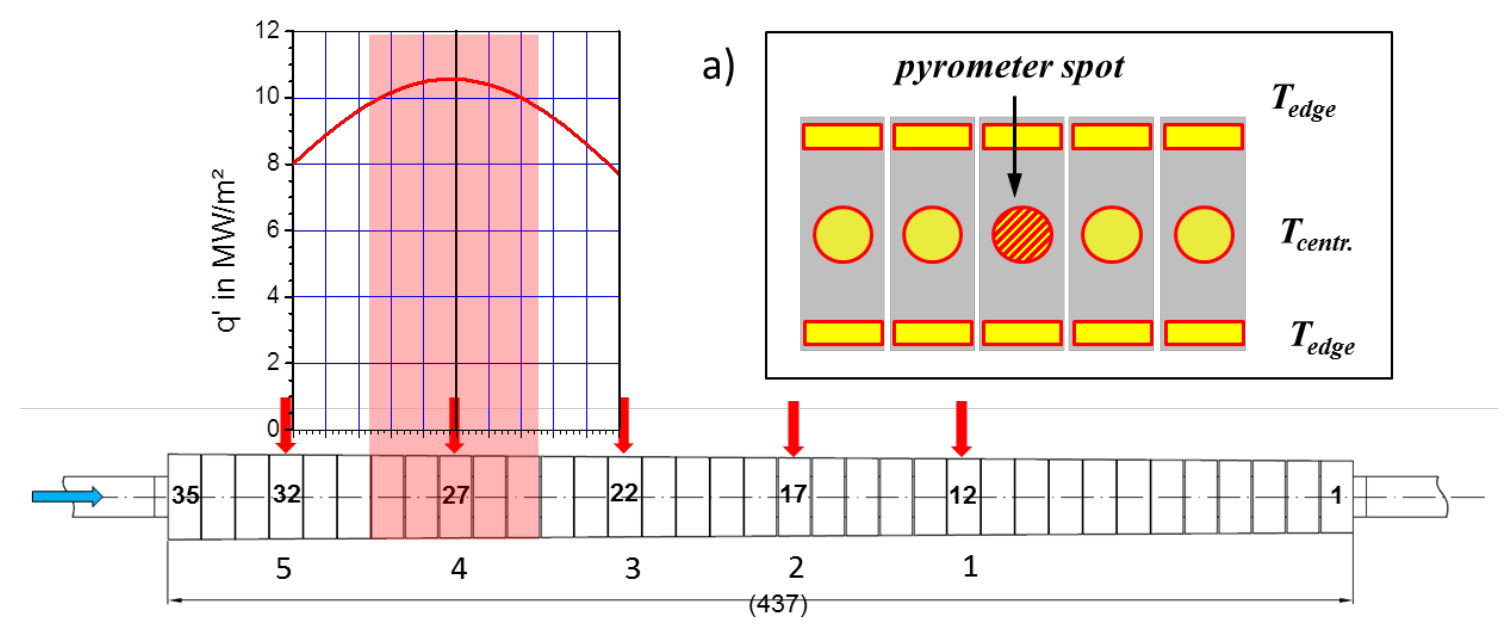

Fig. 2: Arrangement of the five HHF test positions marked with the red arrows. Numbering of the $W$ monoblocks starts on the right hand side of the component. The heat flux distribution is shown for test position 4 as an example. The insert a) shows the pyrometer spot and the regions of interest (ROIs) 
$T_{\text {edge }}$ and $T_{\text {centre }}$ for the IR analysis of surface temperature evaluation as described more in detail section 4.2 and 4.3 .

\section{Results and discussion of surface temperature measurements}

For the surface temperature measurements a two-colour pyrometer $(\lambda \sim 1.6 \mu \mathrm{m}$, $\varnothing 8 \mathrm{~mm}$ spot size, temperature range $\left.500-1700^{\circ} \mathrm{C}, 1 \mathrm{kHz}\right)$ [6] and an IR camera Infratec VARIOCAM HD $(\lambda \sim 10 \mu \mathrm{m}, 12$ $\mathrm{Hz}$ ) were used.

\subsection{Pyrometer measurements}

The pyrometer was focussed on the centre of the central block as shown in Fig. 2. In two-colour pyrometry the necessity of knowing the emissivity of the analysed surface is eliminated by using the intensity ratio measured in two different wavelength bands. This is, however, only fully correct for a so-called "grey body", where the emissivity is assumed to be independent of wavelength and temperature. This is surely not the case for tungsten as numerous literature data show, see e.g. [7]. For the emissivity correction we used data from reference [77] measured on tungsten at $1200 \mathrm{~K}$ surface temperature. The monochromatic emissivity correction was performed according to the literature [8]. The summary of the pyrometrical temperature measurements for all components and test positions is given in Table 1. The measured temperatures are in a good agreement with the FEM calculated temperatures, no significant temperature increase was detected. The two components delivered by JADA show slightly higher values, however within the expected temperature range. Fig. 3. summarizes the individual temperature traces on a selected test position. We show these data because block no. 27 is close to an expected outer strike point in WEST where the maximal heat flux is predicted as published ( Fig.4 in [9]).

\begin{tabular}{|l|c|cc|cc|cc|cc|cc|}
\hline Component & No of cycles on & \multicolumn{2}{|c|}{ Pos. 1, $\mathbf{T}_{\text {pyrom }}\left[{ }^{\circ} \mathbf{C}\right.$ ] } & \multicolumn{2}{|c|}{ Pos. 2, $\mathbf{T}_{\text {pyrom }}\left[{ }^{\circ} \mathbf{C}\right.$ ] } & \multicolumn{2}{|c|}{ Pos. 3, $\mathbf{T}_{\text {pyrom }}\left[{ }^{\circ} \mathbf{C}\right.$ ] } & \multicolumn{2}{|c|}{ Pos. 4, $\mathbf{T}_{\text {pyrom }}\left[{ }^{\circ} \mathbf{C}\right]$} & \multicolumn{2}{|c|}{ Pos. 5, $\mathbf{T}_{\text {pyrom }}\left[{ }^{\circ} \mathbf{C}\right.$ ] } \\
& each position & start & end & start & end & start & end & start & end & start & end \\
\hline WCAT-001 & 100 & 853 & 867 & 863 & 873 & 877 & 868 & 866 & 858 & 859 & 849 \\
WCAT-002 & 100 & 848 & 859 & 850 & 865 & 872 & 876 & 866 & 859 & 868 & 854 \\
WCAT-003 & 100 & 875 & 894 & 873 & 887 & 893 & 901 & 897 & 895 & 884 & 879 \\
WCAT-004 & 100 & 869 & 899 & 828 & 857 & 857 & 880 & 839 & 852 & 846 & 848 \\
WJKA-004 & 10 & & 895 & & 917 & & 910 & & 929 & & 917 \\
WJMI-002 & 10 & & 909 & & 919 & & 925 & & 942 & & 950 \\
\hline
\end{tabular}

Table 1: The table summarizes the pyrometrically measured temperatures of the centre blocks; start temperature corresponds to pulse \#10, end temperature to \#100, excepted WJKA-004 and WJMI-002. The detailed time evolution of the column marked in grey is shown in Fig. 3. 


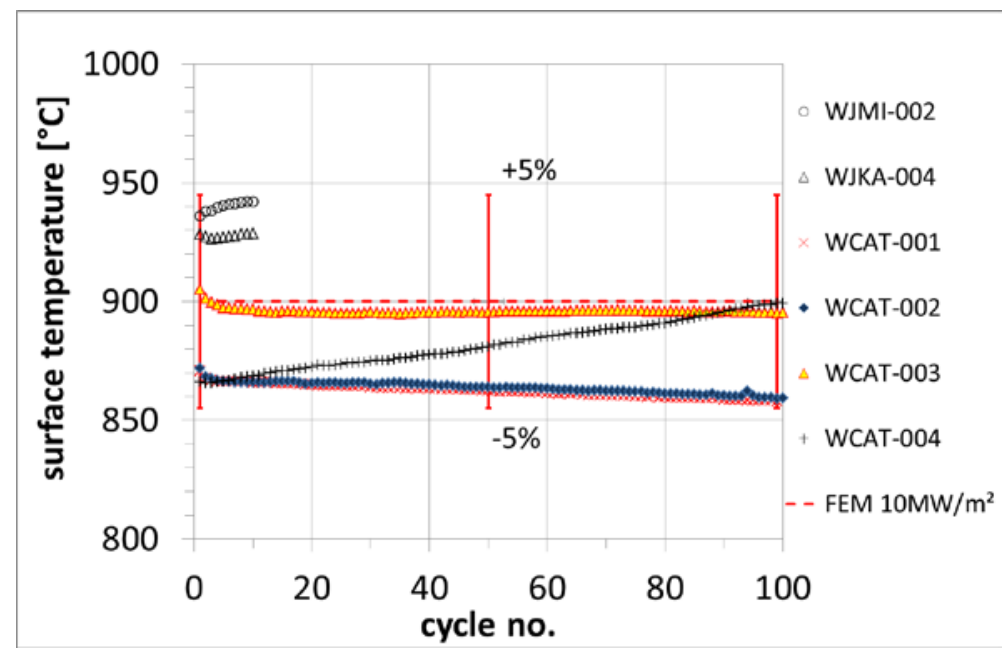

Fig. 3: Summary of pyrometrically measured steady-state surface temperatures of the $10 \mathrm{MW} / \mathrm{m}^{2}$ loaded W block no. 27 for all components. This position is marked in Fig.2. Note, the components WJMI-002 and WJKA-004 were tested with 10 cycles only. The presented FEM calculation was performed for the GLADIS cooling conditions described in sec.3.1.

\subsection{IR measurements of surface temperature distribution}

The main challenge of a reliable IR measurement on $\mathrm{W}$ is the high variability of the emissivity $\varepsilon$. The emissivity can be very different from component to component and also vary with pulse number. $\varepsilon$ depends strongly on the machining quality and in the range of long-wavelength IR also on the surface temperature itself. For the latter see e. g. reference [10]. During the cyclic heat loading a cleaning process of the surface due to evaporation or reduction of $W$ oxides influences strongly $\varepsilon$. The application of a fixed uniform $\varepsilon$ value is not possible for a reliable IR assessment of a test campaign. Therefore we devised a two-colour pyrometer based temperature correction algorithm for each cycle to extract useful temperature values from the IR raw data [11].

\subsubsection{Transient temperature development}

Measurements of the transient evolution of centre temperature of the loaded blocks were performed at the end of cycling. The comparison of the calculated and measured transient behaviour of the $\mathrm{W}$ blocks allows a temperature dependent validation of the material data and heat transfer coefficients employed in FE modelling. In Fig. 4 is shown an example of the transient surface temperature development during a $10 \mathrm{MW} / \mathrm{m}^{2}$ pulse. 


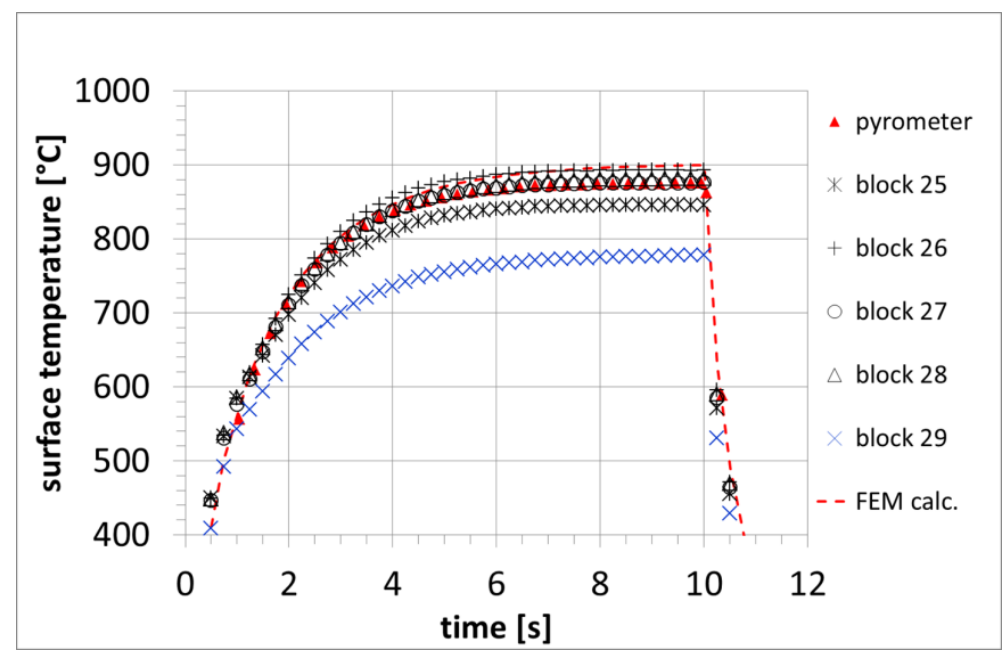

Fig. 4: Transient surface temperature development during a $10 \mathrm{MW} / \mathrm{m}^{2}$ pulse. The example shows the temperatures of the block centre of the last heat pulse on WCAT-003, test position 4, measured by IR camera. Due to the shadow of the beam scraper the temperature of block 29 is slightly lower.

\subsubsection{Steady-state temperature distribution during cycling}

A local or global increase of the monoblock surface temperature during cycling is the result of a degradation of the heat transfer through the component. Due to the strong mismatch of the thermal expansion of $\mathrm{W}$ and the CuCrZr cooling tube, high thermally induced stresses occur in the $\mathrm{W} / \mathrm{Cu}$ interface and could induce a progressive degradation of the bonding quality. The pixel-wise analysis of the steady-state temperature distribution for each block and each cycle allows the detection of growing hot spots due to bonding defects. Furthermore, the analysis of the temperature evolution of the two outer block edges compared to the block centre eliminates slight fluctuations of the loading or cooling conditions. The geometrical arrangement of these ROIs is shown for the assessed blocks in Fig. 2a. The applied assessment criterion $\Delta \mathrm{T}_{\mathrm{N}}$ describes the development of the difference between regions of interest (ROIs) at the block centre and the outer edges during N=100 cycles. An introduction to this method and the performed image processing is published in reference [12].

$$
\Delta T_{N}=\left(T_{\text {edge }}-T_{\text {centre }}\right)_{N}-\left(T_{\text {edge }}-T_{\text {centre }}\right)_{1}
$$

The histogram of $\Delta \mathrm{T}_{100}$ of all tested $\mathrm{W}$ monoblocks generates the input for the statistical analysis of the manufacturing quality.

\subsection{Statistical evaluation as quality assessment}

A stable manufacturing process of a high number of components gives typically products whose measurable properties correspond to a Gaussian distribution. Two parameters, $\mu$ (mean) and $\sigma$ 
(standard deviation), describe the Gaussian distribution. To assess the variability in the manufacturing quality of the PFUs supplied by ASIPP, a statistical assessment of all 96 blocks loaded with 100 cycles at $10 \mathrm{MW} / \mathrm{m}^{2}$ has been performed. A Gaussian with $\mu=-0.4 \pm 0.6$ and $\sigma=8.8 \pm 0.6$ fits the $\Delta \mathrm{T}_{100}$ histogram of 192 samples well. In this state, the data are not sufficient to describe the thermal performance of the WEST PFUs at higher heat loads and pulse numbers. Such data are not available, therefore we show another set of intensively tested components from other suppliers (up to 500 cycles at $20 \mathrm{MW} / \mathrm{m}^{2}$ ) for comparison [11]. The $\Delta \mathrm{T}_{100}$ distribution of these similar $\mathrm{W}$ monoblock components is marked with blue asterisks in Fig. 5.

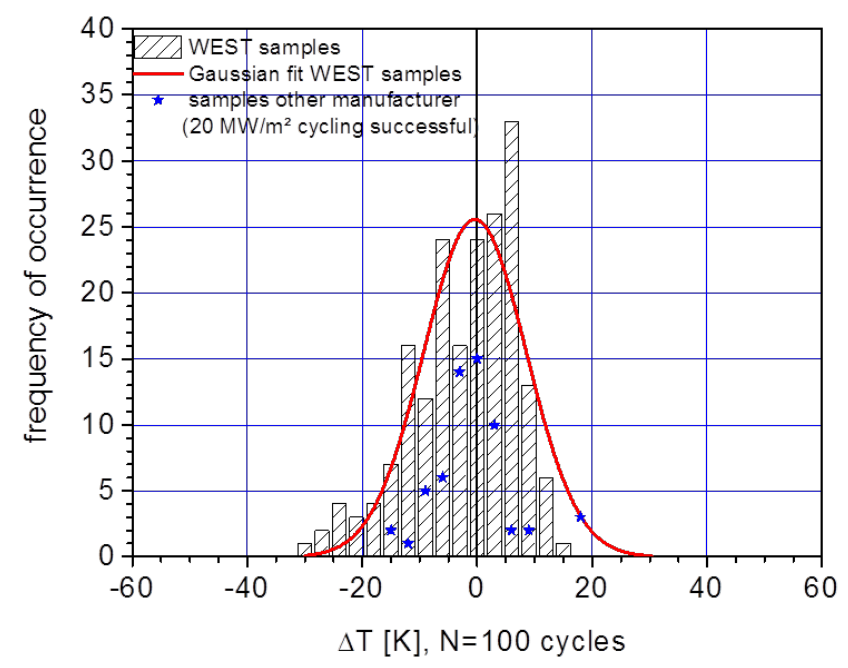

Fig. 5: Histogram of measured $\Delta \mathrm{T}_{100}$ for all $10 \mathrm{MW} / \mathrm{m}^{2}$ tested $\mathrm{W}$ monoblocks of the four ASIPP components as well as data from tests of similar components from other suppliers for comparison.

For the quality assessment of the WEST PFUs we can state:

1. A Gaussian of $\Delta \mathrm{T}_{100}$ is an indication for a reproducible manufacturing of the PFUs because there are no strong excursions of the data.

2. $\mu$ centered about zero indicates a stable thermo-mechanical behavior without increasing degradations of the W/CuCrZr interfaces. Negative $\Delta \mathrm{T}_{100}$ could be explained by the fact that the stress concentration in the W/CuCrZr interface preferentially occurred at the thermally highest loaded part of the cooling tube. This results in an increase of the central W block temperature during cycling. It is an indication that the thermal strain in the $\mathrm{W} / \mathrm{CuCrZr}$ interface during a $10 \mathrm{MW} / \mathrm{m}^{2}$ loading cycle is not fully reversible.

3. The distribution obtained for the WEST PFUs supplied by ASIPP (WCAT-001 - 004) is rather similar to the distribution of intensively HHF tested W monoblock components from other suppliers: both are symmetric and centered around zero. 


\section{Results and conclusions}

Six WEST PFUs prototypes of different suppliers have been HHF tested in GLADIS prior to the installation of the WEST platform. The HHF test results confirm the non-destructive examinations performed by the manufacturer and CEA, no defects of the components were detected.

The surface temperature analysis after 100 cycles at $10 \mathrm{MW} / \mathrm{m}^{2}$ of the four ASIPP PFUs yields a Gaussian distribution centred around zero. This indicates reproducible manufacturing processes which result in components with a uniform quality. Due to time restriction, this analysis was not performed for the JADA prototypes.

A quantitative assessment of the prototypes with respect to the performance under higher heat loads or higher cycle numbers would require further HHF testing.

\section{Acknowledgement}

This work has been partially carried out within the framework of the EUROfusion Consortium (WP PFC) and has received funding from the Euratom research and training programme 2014-2018 under grant agreement No 633053.

\section{References:}

\footnotetext{
${ }^{1}$ A. Grosman et al., Fusion Engineering and Design 88 (2013) 497-500

2 J. Bucalossi et al., Fusion Engineering and Design 89 (2014) 907-912

${ }^{3}$ H. Greuner et al., Journal of Nuclear Materials 367-370 (2007) 1444

${ }^{4}$ T. Hirai, et al., Examinations for Leak Tightness of Actively Cooled Components in ITER and Fusion Devices, submitted to Physica Scripta

${ }^{5}$ H. Greuner et al., Fusion Engineering and Design 82 (2007) 1713-1719

${ }^{6}$ Dr. Georg Maurer GmbH, Kohlberg, Germany, type QKTD 1075

${ }^{7}$ Y. S. Touloukian, D.P. DeWitt, 1970 Thermophys. Prop. of Matter 7796

${ }^{8}$ Vollmer M and Möllmann K.-P. Infrared Thermal Imaging, 2010, Wiley-VCH , Weinheim

${ }^{9}$ P. Languille et al., Fusion Engineering and Design 109-111 (2016) 294-298

${ }^{10}$ J. C. de Vos, Physica 20 (1954) 690

${ }^{11}$ H. Greuner et al., article in press, http://dx.doi.org/10.1016/j.fusengdes.2017.02.092

${ }^{12}$ H. Greuner et al., Phys. Scr. T138 (2009) 014063
} 